\title{
Optimization of Turbine Engine Cycle Analysis with Analytic Derivatives
}

\author{
Dr. Tristan Hearn, ${ }^{*}$ Eric Hendricks, ${ }^{*}$ Jeffrey Chin, ${ }^{*}$ Justin Gray, ${ }^{\dagger *}$ Dr. Kenneth T. Moore,${ }^{\ddagger}$
}

\begin{abstract}
A new engine cycle analysis tool, called Pycycle, was built using the OpenMDAO framework. Pycycle provides analytic derivatives allowing for an efficient use of gradient-based optimization methods on engine cycle models, without requiring the use of finite difference derivative approximation methods. To demonstrate this, a gradient-based design optimization was performed on a turbofan engine model. Results demonstrate very favorable performance compared to an optimization of an identical model using finite-difference approximated derivatives.
\end{abstract}

\section{Introduction}

Design of an aircraft is a complex, coupled, and increasingly multi-disciplinary problem. There are a large number of potential physical design variables to vary, and tremendous number of operating conditions to consider. Some of these conditions primarily impact the design objective, while others principally impose constraints on this objective. An optimal configuration, particularly in the case of tight inter-disciplinary coupling, is often not intuitive. Given this, it is no surprise that numerical optimization has become a mainstream tool in conceptual design.

Numerical optimization algorithms can generally be classified into two categories, gradient-based and gradient-free. Gradient-based optimization methods use derivatives of a model to iteratively perturb parameter values in a direction which improves the value of an objective function until a terminating criteria is reached (typically based on the KKT optimality conditions). This contrasts with gradient-free methods, which may use a variety of sampling and local search strategies to determine optimal parameter values for a model. ${ }^{1}$ There are practical advantages and disadvantages to both types of optimization algorithms. Broadly speaking, gradient-based optimization, though it is susceptible to convergence onto local optima, uses far less function evaluations compared to gradient-free methods. ${ }^{2}$ For large-scale computationally-expensive models, this is a significant advantage, particularly when combined with adjoint methods. As the name implies, gradient-based optimization algorithms rely on gradient information to achieve their reduced computational cost.

Finite-difference approximation is often used to compute derivatives, but this method scales poorly as the number of design variables increases. Finite-difference approximations are also sensitive to the step size used to estimate them, especially when a model has one or more internal solvers converging a nonlinear system of equations. For these reasons, models which provide analytic derivatives in addition to their analysis outputs are particularly suitable for efficient optimization with gradient-based methods.

There are an increasing number of aircraft design tools that do provide one or more forms of analytic derivatives for the purpose of gradient-based optimization. Many CFD and FEA codes now come with adjoint analytic derivative capabilities. ${ }^{3-6}$ Recent work has begun on an adjoint-based aircraft optimization to perform aircraft mission analysis as well, ${ }^{7}$ including a a new mission analysis tool, named PyMission, that includes adjoint derivatives for trajectory optimization. ${ }^{8}$ However, to date, there exists no propulsion analysis tool that provides analytic derivatives. The current state of the art for propulsion analysis is the Numerical Propulsion System Simulation (NPSS) tool. ${ }^{9}$ This tool provides a general framework to model a very wide variety of thermodynamics cycles, from simple turbojets to complex co-generation cycles. ${ }^{10,11}$ Despite the effectiveness of NPSS as an analysis tool, it does present some challenges when being used

*Aerospace Engineer, Propulsion Systems Analysis Branch

${ }^{\dagger}$ Doctoral Pre-Candidate, Department of Aerospace Engineering, University of Michigan

${ }^{\ddagger}$ Senior Systems Engineer, Propulsion Systems Analysis Branch 
in an optimization context. Geiselhart et. al. used NPSS as to model the propulsion system of a lowboom supersonic transport, but noted numerical stability as a primary motivation for using gradient free optimization methods. ${ }^{12}$ Hendricks et. al. highlighted acute inaccuracy of finite difference gradients when optimizing a turbine design, using a mean-line turbomachinery model built in NPSS. ${ }^{13}$ To address these challenges, a new cycle analysis tool, Pycycle, was written using OpenMDAO. Previous work presented a validation of the underlying thermodynamics modules in PyCycle. ${ }^{14}$ In this paper we present the application of PyCycle to the analysis and optimization of a complete on-design turbofan engine cycle.

This paper is organized as follows. Section A provides a more in-depth description of how analytical derivatives, which are provided within disciplinary components of a model, can be used to compute total derivative quantities required by a gradient-based optimizer. Section B describes the structure of the Pycycle and how it differs conceptually from existing engine cycle tools (with supplementary graphics provided in Section V). Section II formulates the example turbofan engine optimization problem. Section III reports the results of applying gradient-based optimization to an implementation of this problem created using Pycycle. The performance of this optimization is compared across several metrics to optimizations performed on identical models, but using finite-difference derivatives. Finally, comments and future applications are discussed in Section IV.

\section{A. Model Optimization With Analytic Derivatives}

Following the methodology of Martins et. al, ${ }^{15}$ it is informative to first illustrate how discipline-level derivative information can be used to compute total derivative quantities across a model (even in the presence of tight inter-disciplinary coupling). Let

$$
\mathbf{F}(\mathbf{x}, \mathbf{y}): \mathbb{R}^{k+n} \rightarrow \mathbb{R}^{m}
$$

represent the objective function and all constraints of an MDAO problem, with $\mathbf{x} \in \mathbb{R}^{k}$ being a vector of $k$ design variables, and $\mathbf{y} \in \mathbb{R}^{n}$ being a vector of $n$ state variables, returning $\mathbb{R}^{m}$ where $m$ is the total number of constraints plus the objective. Figure 1 illustrates an example model possessing this structure, with data passed between three discipline-level modeling components, $C_{1}, C_{2}$, and $C_{3}$. Here, the components $C_{1}$ and $C_{2}$ are coupled together in a manner that must be solved numerically.

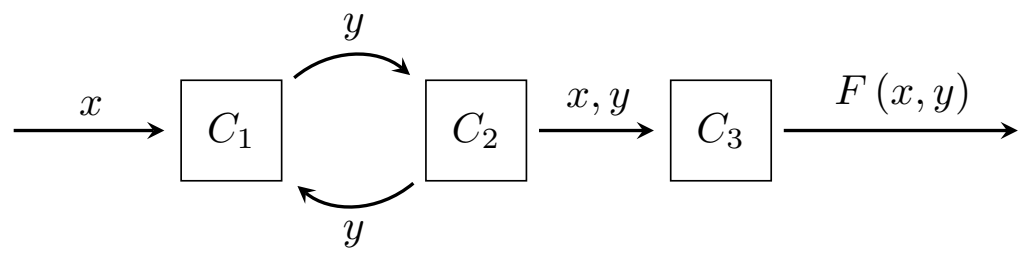

Figure 1. Illustration of a coupled model, with input variables $x$, coupling variables $y$, and output $F(x, y)$.

Next, let

$$
\mathbf{R}(\mathbf{x}, \mathbf{y}): \mathbb{R}^{k+n} \rightarrow \mathbb{R}^{n}
$$

represent a series of $n$ residual equations that describe the multidisciplinary coupling between $C_{1}$ and $C_{2}$, such that for a feasible set of values for $\mathbf{x}, \mathbf{y}$, (i.e., when data passes from discipline components $C_{2}$ to $C_{3}$ ) we have

$$
\mathbf{R}(\mathbf{x}, \mathbf{y})=0
$$

To compute a gradient for an optimization iteration we will need to compute the derivative of the quantities of interest $\mathbf{F}$ with respect to the design variables $\mathbf{x}$,

$$
\underbrace{\frac{d \mathbf{F}}{d \mathbf{x}}}_{m \times k}=\underbrace{\frac{\partial \mathbf{F}}{\partial \mathbf{x}}}_{m \times k}+\underbrace{\frac{\partial \mathbf{F}}{\partial \mathbf{y}}}_{m \times n} \underbrace{\frac{d \mathbf{y}}{d \mathbf{x}}}_{n \times k} .
$$

The residual and state equations may be combined to give an expression for the elements of the total derivative matrix in terms of partial derivatives of the objective functions, constraints, and residual equations. ${ }^{15}$ In plain terms, the total derivative matrix is constructed one column at a time (known as the forward form) as 


$$
\underbrace{\frac{d \mathbf{F}}{d \mathbf{x}_{i}}}_{m \times 1}=\underbrace{\frac{\partial \mathbf{F}}{\partial \mathbf{x}_{i}}}_{m \times 1}-\underbrace{\frac{\partial \mathbf{F}}{\partial \mathbf{y}}}_{m \times n} \underbrace{\left(\frac{\partial \mathbf{R}}{\partial \mathbf{y}}\right)^{-1} \frac{\partial \mathbf{R}}{\partial \mathbf{x}_{i}}}_{n \times 1}
$$

for each $\mathbf{x}_{i}$. Note that $(\cdot)^{-1}(\cdot)$ does not necessarily denotes a actual matrix inversion, and could instead be the numerical solution of a linear system by a suitable numerical method (either direct or iterative). Indeed, in a large distributed model the matrix $\frac{\partial \mathbf{R}}{\partial \mathbf{y}}$ may not be explicitly assembled at all, making iterative methods such as GMRES ${ }^{16}$ a good choice for solving the linear system in Equation 5.

Similarly, the adjoint form of the derivatives may be computed as

$$
\underbrace{\frac{d \mathbf{F}_{i}}{d \mathbf{x}}}_{1 \times k}=\underbrace{\frac{\partial \mathbf{F}_{i}}{\partial \mathbf{x}}}_{1 \times k}-\underbrace{\left(\left(\frac{\partial \mathbf{R}^{T}}{\partial \mathbf{y}}\right)^{-1} \frac{\partial \mathbf{F}_{i}^{T}}{\partial \mathbf{y}}\right)^{T}}_{1 \times n} \underbrace{\frac{\partial \mathbf{R}}{\partial \mathbf{x}}}_{n \times k},
$$

for each $\mathbf{F}_{i}$. In other words, if the Jacobian matrices $\frac{\partial \mathbf{F}}{\partial \mathbf{x}}, \frac{\partial \mathbf{F}}{\partial \mathbf{y}}, \frac{\partial \mathbf{R}}{\partial \mathbf{y}}$, and $\frac{\partial \mathbf{R}}{\partial \mathbf{x}}$ can be provided within the model, then the total derivatives can be computed efficiently without re-evaluation of the non-linear model. OpenMDAO collects partial derivatives provided by individual model components and automatically aggregates them to solve for the total derivative across a multidisciplinary model. Model components which do not provide any derivative information can individually have their derivatives approximated using finitedifference, allowing for an OpenMDAO model to automatically make use of a mix of analytic and finitedifference derivatives when total derivative quantities are needed.

\section{B. Structure Of Pycycle}

Pycycle is a new thermodynamic cycle analysis tool developed using the OpenMDAO framework. Like NPSS, Pycycle provides a means to perform single dimensional (1D) steady-state engine cycle analysis, based on the numerical convergence of equilibrium chemistry relations. For a cycle analysis performed in "on-design" mode, the initial engine cycle reference (or "design" point calculation) used to size engine geometry can be viewed as a four-step process. To begin, the equilibrium composition of the working fluid is computed by solving a system of nonlinear equations to minimize a Gibbs free energy function. Next, the thermodynamic state variables (temperature $T$, pressure $P$, density $\rho$, entropy $S$, and enthalpy $h$ ) of the fluid mixture is computed. The implementation of these first two steps in Pycycle are outlined in detail in previous works by Gray et al. ${ }^{14}$ Third, the analysis of each thermodynamic cycle stage (the corresponding to physical engine elements) is executed, and the corresponding velocities and areas are calculated. Finally, the system is brought to convergence by varying engine geometry and operating parameters until all boundary conditions reach agreement, and power imbalances are driven to zero (in the case of steady-state) or to an timedependent integrated state (in the case of transient). Following the successful convergence of an on-design reference case, a single or series of "off-design" cases may then be computed. In this mode, elements sized using the reference on-design case compute their operating characteristics at a specified flight Mach number, throttle setting, and altitude. Aggregating the results from a sufficiently large number of off-design cases allows an analyst to identify design choices which are sufficiently robust over a large range of operational flight conditions. ${ }^{9}$

From a high level, the design of Pycycle was heavily influenced by NPSS. Like NPSS, Pycycle uses a heavily object-oriented structure to represent the physical components (typically referred to as elements) of an engine cycle. Object orientation is an inherent aspect of models built within OpenMDAO, as it provides a reasonable encapsulation of separable calculations, while enabling a modular data flow representation of an overall model. As illustrated in Section A, is this model component compartmentalization that allows for analytical total derivatives (in either the forward or adjoint formulation) to be implemented, tested in a user-friendly and highly maintainable fashion. Aside from the addition of analytical derivatives to the chemical equilibrium and engineering calculations, the main difference between Pycycle and NPSS is in how the required thermodynamics calculations are organized within their respective elements. In Pycycle, thermodynamic chemical equilibrium calculations (and the resulting flow properties) may be handled in their own components, or exposed directly to the framework. This contrasts with NPSS, where thermodynamic 
calculations were strictly contained inside the the individual engine elements and are thus generally hidden from the rest of framework.

There are practical advantages to having the flexibility to treat the thermodynamic calculations on the same level as the engineering calculations. For instance, the derivative calculations can be compartmentalized, and therefore implemented and tested more easily. This also allows for some of these variables to be converged at a higher level, or even handed to an optimization algorithm to perform simultaneous analysis and design. ${ }^{17}$

For reference, Section V provides an illustration of each of the Pycycle engine elements which were used to construct the turbofan engine model for the optimization described in the following sections.

\section{Application to a gradient-based optimization}

To demonstrate the advantage of the analytic derivatives provided by Pycycle, a simple optimization problem was set up in such a way that a direct comparison to an NPSS-based optimization would be possible.

For this, a single point on-design turbofan engine model was constructed within both Pycycle and NPSS. Figure 2 illustrates the structure of this model. Generally speaking, this is a type of open Brayton engine cycle with a core stream passing through a fan, compressor, burner, and turbines, with a second stream of air compressed by the fan but bypassing the core. There are a number common design parameters to most turbofan engines which may be selected by an engine cycle analyst. These are the overall pressure ratio $(\mathrm{OPR})$, combustor temperature $\left(T_{4}\right)$, ratio of mass flow between the bypass and core streams (BPR), and pressure ratios of the fan and engine compressor (FPR and CPR). These variables have the greatest impact on the typical quantities of interest, such as thrust-specific fuel consumption (TSFC). ${ }^{18}$

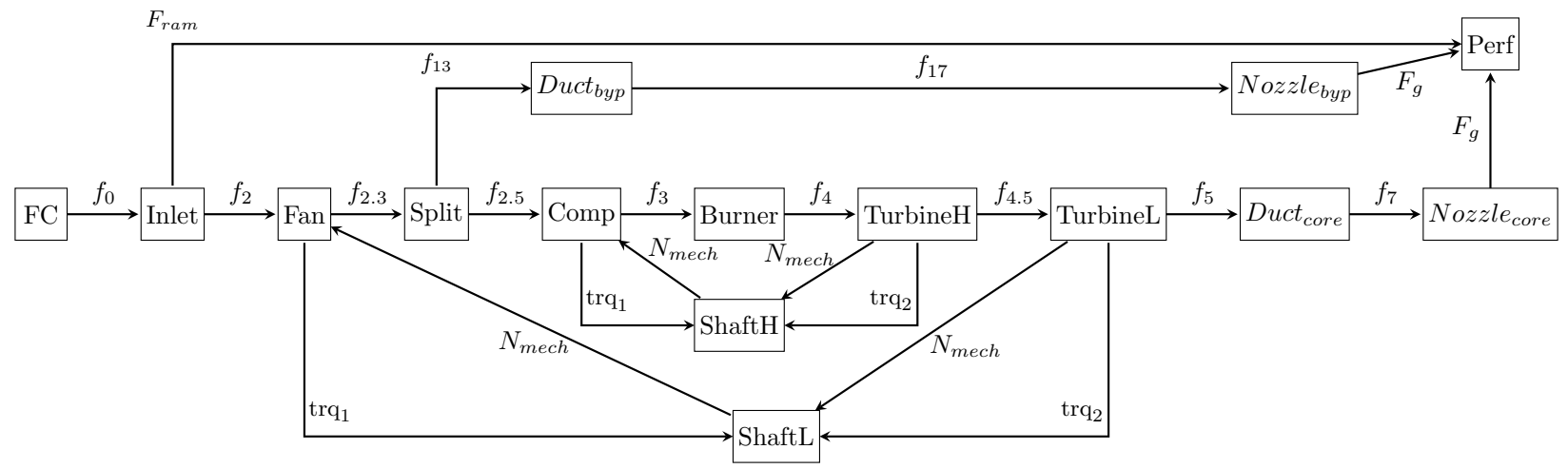

Figure 2. Directed-graph structure of a turbofan engine model. Each rectangle represents an analysis component, and the arrows represent data relationships and required executing order. Each label represents a variable or series of variables being passed between the elements (e.g. $f_{i}$ represents a flowstation, carrying a series of variables representing computed flow properties.)

As mentioned in Section B, each element (such as a compressor or turbine) needs to compute the total and static flow properties at its exit boundaries (and sometimes at interior points) by satisfying the steadystate thermodynamic equilibrium equations. For most elements, this involves an iterative solution of the thermo equations coupled with some element-specific performance and sizing calculations. At a higher level in the model, the power on the two shaft spools must be properly balanced by varying the pressure ratio of the low-pressure turbine (LPT) and high-pressure turbine (HPT) so that the net power is zero (no acceleration.) This introduces a cyclic containing the compressor, HPT, LPT, and shaft elements. When this coupling is in a properly converged state, the quantities $\mathrm{Shaft}_{\text {net pwr. }}$ and $\mathrm{Shaft}_{\mathrm{net}}$ pwr. are each zero. This is an implicit condition that is satisfied using an analytic derivative based Newton-Krylov solver built into OpenMDAO and utilized in Pycycle, and a Newton-Raphson (using finite-difference jacobian approximations with Broyden updates) solver within NPSS. ${ }^{19}$ For both the NPSS and Pycycle models, the non-linear solvers were configured to have a tolerance of $10^{-6}$.

An optimization problem was then formulated to minimize thrust-specific fuel consumption (TSFC), with respect to fan pressure ratio (FPR), compressor pressure ratio (CPR), bypass ratio (BPR), and mass-flow rate $(W)$. Engine burner temperature $\left(T_{4}\right)$ was constrained to be less than 3000 degrees Rankin, and a net 
thrust $\left(F_{n}\right)$ target of 25,000 lbf was set. Overall pressure ratio (OPR) was also constrained to be equal to 30. The optimization problem therefore has the formulation shown in Table 1.

Minimize:

\begin{tabular}{|c|c|c|c|}
\hline & & TSFC & \\
\hline With respect to: & & & \\
\hline & $1 \leq$ & FPR & $\leq 2$ \\
\hline & $1 \leq$ & CPR & $\leq 30$ \\
\hline & $1 \leq$ & BPR & $\leq 12$ \\
\hline & $1 \leq$ & $\mathrm{W}$ & $\leq 2000 \frac{\mathrm{lbm}}{\mathrm{s}}$ \\
\hline Such That: & & & \\
\hline & & OPR & $=30$ \\
\hline & & $F_{n}$ & $=25,000 \mathrm{lbf}$ \\
\hline & & $T_{4}$ & $\leq 3000^{\circ} \mathrm{R}$ \\
\hline
\end{tabular}

Table 1. The formulation of the turbofan optimization problem.

\section{Results}

The optimization problem was solved using the $\mathrm{SNOPT}^{20}$ optimizer from within OpenMDAO. The identically defined NPSS turbofan model was also optimized using SNOPT via an OpenMDAO wrapper, using derivatives approximated via forward-form finite-difference. Three separate wrapped optimizations were performed on this NPSS implementation, with step sizes of $10^{-5}, 10^{-4}$ and $10^{-3}$. An optimality tolerance of $10^{-3}$ on the objective function and feasibility tolerance of $10^{-5}$ on the constraints was specified to the SNOPT optimizer for both optimizations. Table 2 compares the parameter and objective values between the baseline and optimized configurations, for both turbofan model implementations. The baseline and optimized objective and constraint values computed by the Pycycle model were also verified using the NPSS implementation, as a check on the accuracy of the Pycycle thermodynamic calculations. Broadly speaking, the results in Table 2 show agreement between the Pycycle and NPSS implementations of the on-design turbofan model, as they each achieve nearly the same result when optimized using SNOPT.

From this tabulated data, it is seen that the NPSS-based optimization with a step size of $10^{-5}$ required 120 iterations of the SNOPT optimizer, while the Pycycle optimization required only 44. The number of iterations for step sizes of $10^{-4}$ and $10^{-3}$ was 58 and 52 , respectively. Given that the default step size for finite-difference calculation within OpenMDAO is $10^{-6}$ (and even smaller within optimizers such as SNOPT and SLSQP used alone outside of OpenMDAO), this highlights how computationally difficult even this simple optimization problem would have been without at least some a priori experimentation with step size selection. Note that this observation is only considering main iterations of the SNOPT optimizer, not the additional iterations that would be required for the finite-difference derivative estimation. In contrast, none of these considerations are relevant to the use of Pycycle, thanks to the analytical derivatives provided.

Table 2 also shows that the Pycycle convergence achieved the tightest absolute tolerance on both the internal shaft power balances as well as the three optimization constraints. The NPSS model did consistently achieve a configuration with $1.7 \%$ greater TSFC reduction over baseline, however when the optimization solution achieved by Pycycle is verified afterwards using NPSS, it does agree with the TSFC value of 0.320, indicating a small potential mismatch in the final performance calculations between NPSS and Pycycle, but consistency is the establishment of the parameterization as a feasible minimizing point. The mismatch in mass flow rates, $W$, may be related to this, or due to tighter tolerances achieved on the constraints and internal balances in Pycycle. In any case, the difference in solutions returned by the four optimizations is interpreted to be small enough to consider the results as being in mutual agreement. The three optimizerselected pressure ratios and the internally solved turbine pressure ratios were all very consistent across the four optimizations.

Next, Figure 3 illustrates the logarithm of both the overall SNOPT model feasibility and optimality measures for the Pycycle and NPSS optimizations, as a function of iteration number. These show that both 


\begin{tabular}{crrrrr}
\hline & Baseline & Optimized (Pycycle) & \multicolumn{3}{c}{ Optimized (NPSS) } \\
\hline FD step size & - & - & $10^{-5}$ & $10^{-4}$ & $10^{-3}$ \\
\hline FPR & 1.5 & 2.0 & 2.0 & 2.0 & 2.0 \\
CPR & 10.3 & 15.0 & 15.0 & 15.0 & 15.0 \\
BPR & 5.0 & 12.0 & 12.0 & 12.0 & 12.0 \\
$W$ & 500.0 & 1069.2 & 1032.41 & 1032.39 & 1032.40 \\
\hline TurbL & 1.611 & 9.096 & 9.090 & 9.091 & 9.090 \\
TurbH $_{\mathrm{PR}}$ & 1.935 & 2.356 & 2.356 & 2.356 & 2.356 \\
ShaftL $_{\text {net pwr. }}$ & $1.64 \cdot 10^{-6}$ & $4.530 \cdot 10^{-9}$ & -0.023 & -0.022 & -0.022 \\
ShaftH $_{\text {net pwr. }}$ & $6.11 \cdot 10^{-8}$ & $9.555 \cdot 10^{-9}$ & $2.823 \cdot 10^{-6}$ & $2.825 \cdot 10^{-6}$ & $2.826 \cdot 10^{-6}$ \\
\hline $\mathrm{TSFC}^{-6}$ & 0.612 & 0.331 & 0.320 & 0.320 & 0.320 \\
$F_{n}$ & 13723.128 & 25000.000 & 25000.003 & 24999.649 & 25000.001 \\
$\mathrm{OPR}$ & 15.450 & 30.000 & 30.000 & 30.000 & 30.000 \\
$T_{4}$ & 2762.254 & 2913.355 & 2913.690 & 2913.690 & 2913.690 \\
\hline
\end{tabular}

Table 2. Comparison of design variables, objectives, and constraint values between the baseline and optimized configuration for the two turbofan models.

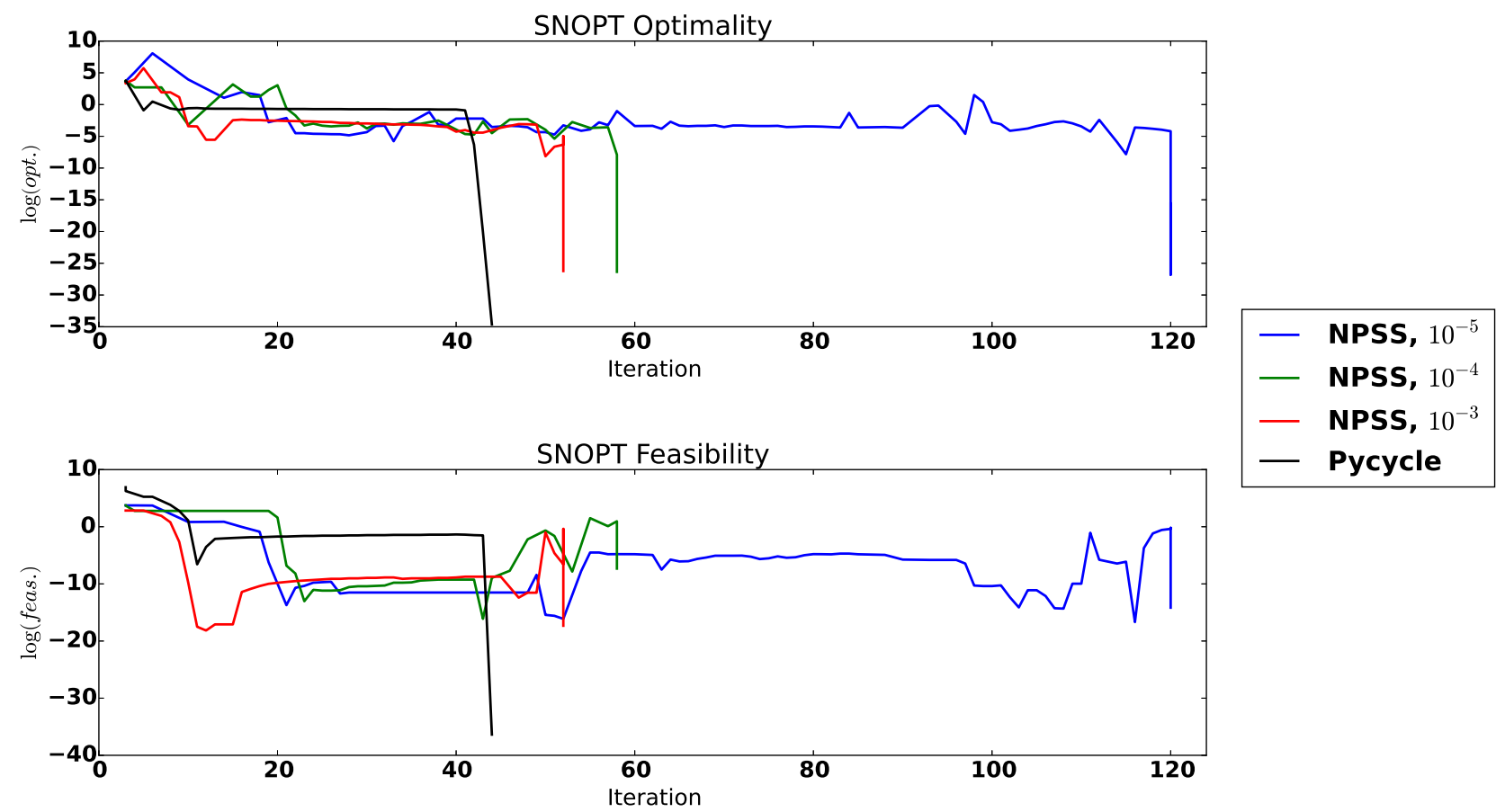

Figure 3. Convergence history of the Pycycle and NPSS turbofan optimization. Here the logarithm of both the feasibility and optimality metrics are plotted with respect to iteration number. 
optimizations experiences a slow evolution from the baseline configuration and then a rapid improvement at a further point in the design space, though the Pycycle optimization reached this point more quickly (at the $44^{\text {th }}$ iteration, noted above). This would indicate that the analytic derivatives provided by Pycycle are precise enough for SNOPT to converge more efficiently in terms of required iterations than for any of the finite-difference driven optimizations. Note also that the evolution of the feasibility and optimality metrics are far smoother for Pycycle than for any of the finite-difference driven optimizations, whose progressions are seen to exhibit far more noise as they progress through the design space. This is interpreted to be due to the precision inherent to the analytic derivatives.

Several summary metrics were also recorded for each optimization, including total number of main iterations, percent improvement in objective function, maximum constraint violation at the optimized configuration, and execution times. These metrics are tabulated in Table 3, and compared with the optimization performed using Pycycle with analytic derivatives. When the total number of model executions are counted (including not just main SNOPT iterations, but executions required for finite-difference approximations), it is seen that the Pycycle based optimization completed within 155 total execution of the turbofan model, while the NPSS-based optimizations required anywhere from 868 to 2,208, depending on the finite-difference step size used. This represents a notably broad range of both computational effort and overall stability of performance in optimizations performed on the turbofan engine model when finite-difference approximations are the only option available. As highlighted before, there is no such careful selection of step size required when optimizing the engine cycle using Pycycle's analytic derivatives.

\begin{tabular}{lcccc}
\hline & Pycycle & \multicolumn{3}{c}{ NPSS } \\
\hline FD step size & - & $10^{-5}$ & $10^{-4}$ & $10^{-3}$ \\
TSFC reduction & $45.9 \%$ & $47.6 \%$ & $47.6 \%$ & $47.6 \%$ \\
Number of SNOPT iterations & 44 & 120 & 58 & 52 \\
Total model executions & 155 & 2208 & 914 & 868 \\
Maximum constraint violation & $3.5 \cdot 10^{-15}$ & 0.003 & 0.351 & $1.2 \cdot 10^{-3}$ \\
Run time (seconds) & 3753 & 30912 & 12796 & 11272 \\
\hline
\end{tabular}

Table 3. Comparison of benchmarks between the Pycycle optimization with analytic derivatives and the NPSS optimization with finite-difference approximated derivatives.

The last row of Table 3 shows total execution time ( or "wall time") required for each of the four performed optimizations. The units of these numbers is seconds, would all appear to be quite large for all four runs considering the rather small scale of the single design point model presented here (representing anywhere from around 1 hour to 8.5 hours). Note that this is due to the platform used to run these optimizations, which was a 64-bit Linux virtual machine running on a native host operating system (Mac OSX 10.10). The virtual OS was required in order to run the wrapped NPSS models, since no native NPSS binaries exist for the Mac OSX platform. Due to the file-wrapped nature of the NPSS model, a fixed-size virtual hard disk was used to limit any latency that might occur from dynamic resizing of the virtual OS's partitions. For consistency, all optimizations of record (including the Pycycle optimization) were performed within this virtual OS. It is expected that if these had instead all been executing on a host OS with greater access to computing resources, that all recorded metrics and conclusions would be identical to what has been discussed thus far in this work, with the exception of a scaling of these recorded times. In addition to the file I/O required for the NPSS-based optimizations, each of the optimizations (including the Pycycle optimization) recorded the execution data from all cases into an on-disk SQLite database, which does introduce some iteration-level cost to execution times as well.

In short, inferences between the relative execution wall times of the Pycycle and NPSS-based optimizations may be more difficult to make than the more direct comparisons of SNOPT iteration requirements and total number of model executions. The Pycycle optimization clearly performed far better than any of the NPSS runs in terms of total execution time, number of iterations required, and fidelity to the model constraints. However, it is worth noting that there is no canonically accepted manner of performing an optimization around an NPSS model. The simple file-wrapped approach used for this work was very straightforward and generalizable, but not carefully designed to improve run-time performance specifically for this 
model. There are a number of possible wrapper implementations that could have favorable run-time behavior relative to the Pycycle optimization, such as a direct in-memory interface, or a file based wrapper that parallelized the executions required for finite-difference gradient estimations. However, improvement of the run-time performance of the NPSS wrapper alone would not change the other results (number of iterations, solution precision, etc.) or conclusions drawn from this work for the comparison of finite-difference to analytic derivatives for engine cycle optimization from a stability or precision standpoint.

Likewise, it can be imagined that in a model sufficiently complex to exhibit variability in finite-difference sensitivity in different areas of the design space, analytic derivatives would be a practical necessity for the increase in stability that they provide.

\section{Conclusion}

This work has presented the application of the thermodynamic cycle analysis tool Pycycle to a gradientbased engine cycle optimization, through the use of analytic derivatives.

Given the favorable results in the use of analytic derivatives demonstrated in Section III, there are a variety of future applications that may be considered. The ability to compute adjoint derivatives across an engine cycle allows for the formulation of multi-disciplinary optimization problems which bridge propulsion to other design disciplines. For instance, an multiple design point (MDP) problem may be formulated to integrate engine cycle analysis with mission analysis and trajectory optimization using analytical adjoint derivatives.

If we consider a use case involving the coupling of engine performance cycle analysis to a more computationally expensive discipline (such as CFD, or trajectory analysis involving a large series of off-design points), we can infer from these results how the availability of analytical adjoint derivatives can enable far more ambitious types of optimization problem formulations than a situation where finite-difference derivatives are the only available option.

\section{Appendix}

Figures 4 through 8 illustrate the structure of the Pycycle engine modeling elements used in the turbofan MDP optimization problem. As per the OpenMDAO 1.0 API, each of the figures collectively represents a Group object, while the rectangular graphics represent Component objects.

More specifically, the rectangular components indicated an engineering calculation, while the elliptical components indicate a chemical equilibrium-based thermodynamic calculation, which is itself is a sub-group illustrated in figure 12. Input parameters and output values are the labels shown without a shape outline.

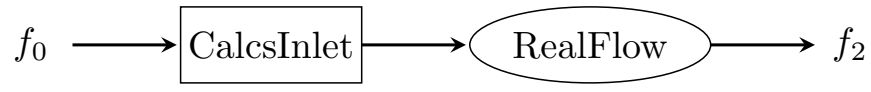

Figure 4. Structure of an inlet element.

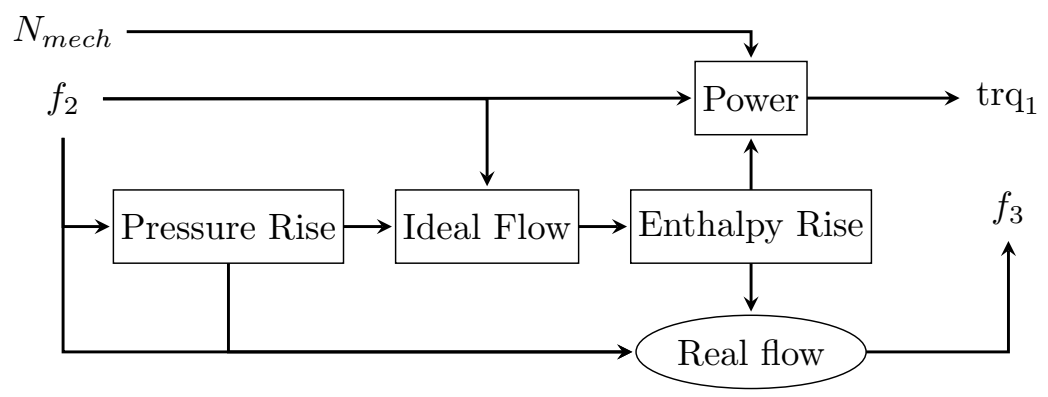

Figure 5. Structure of a compressor element. 


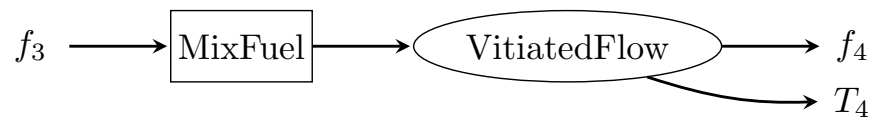

Figure 6. Structure of a burner element.

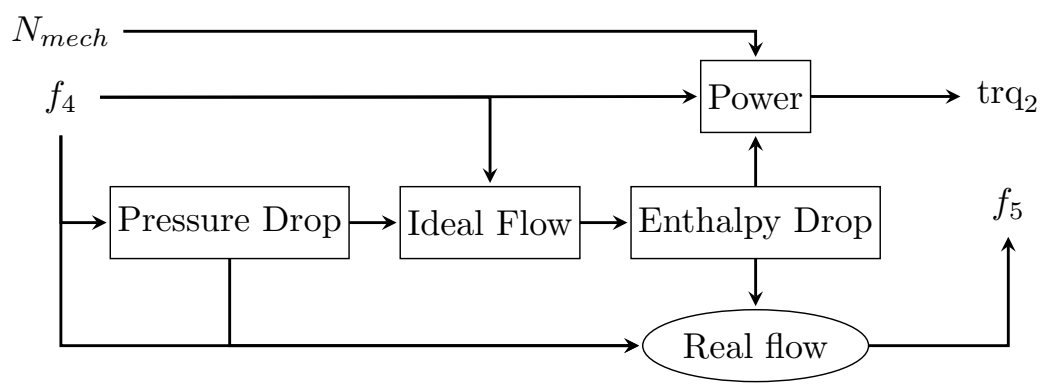

Figure 7. Structure of a turbine element.

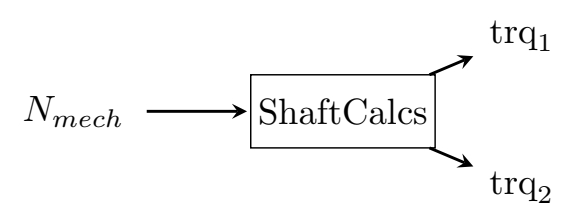

Figure 8. Structure of a shaft element.

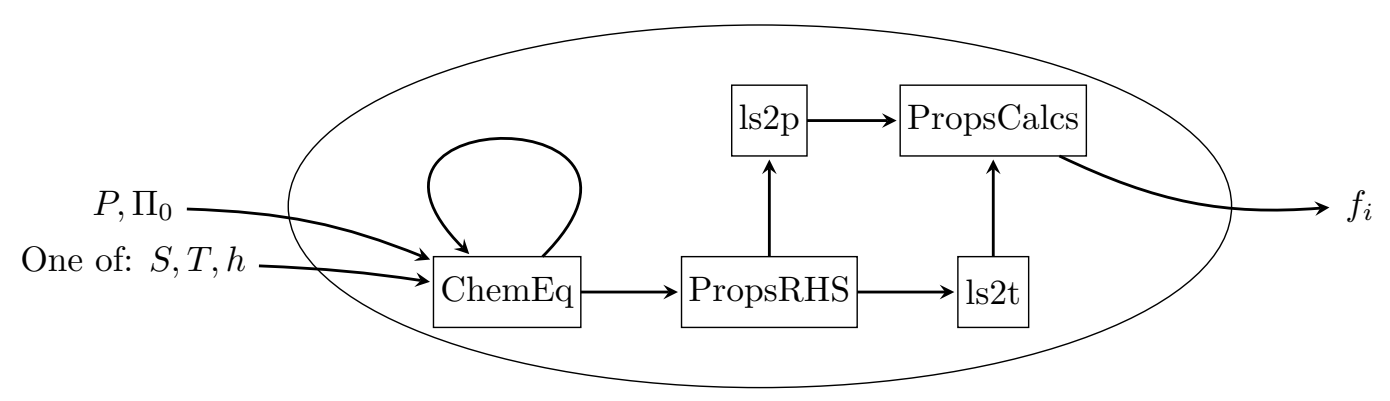

Figure 9. Structure of a thermodynamic chemical equilibrium component.

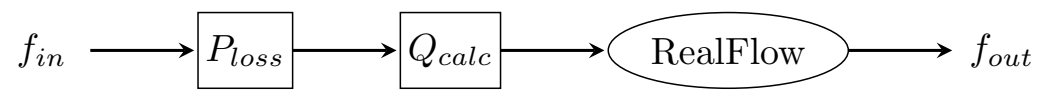

Figure 10. Structure of a duct element.

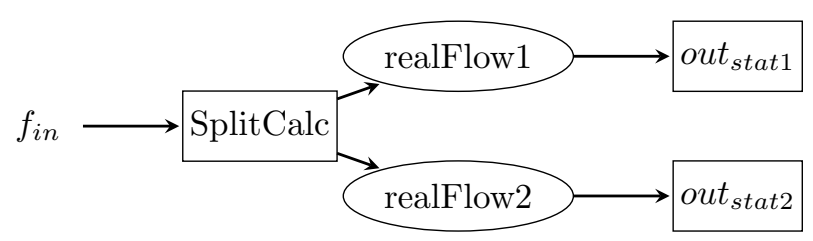

Figure 11. Structure of a splitter element. 


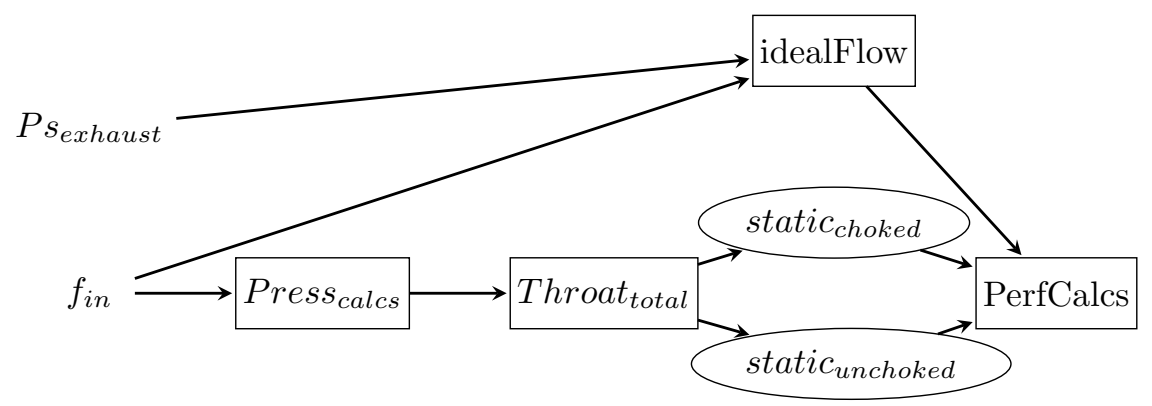

Figure 12. Structure of a nozzle element.

10 of 11

American Institute of Aeronautics and Astronautics 


\section{References}

${ }^{1}$ Rios, L. M. and Sahinidis, N. V., "Derivative-free optimization: a review of algorithms and comparison of software implementations," Journal of Global Optimization, Vol. 56, No. 3, 2013, pp. 1247-1293.

${ }^{2}$ Conn, A. R., Scheinberg, K., and Vicente, L. N., Introduction to derivative-free optimization, Vol. 8, Siam, 2009.

${ }^{3}$ Nielsen, E. J. and Diskin, B., "Discrete Adjoint-Based Design for Unsteady Turbulent Flows on Dynamic Overset Unstructured Grids," AIAA Journal, Vol. 51, No. 6, June 2013, pp. 1355-1373.

${ }^{4}$ Kenway, G. K. W., Kennedy, G. J., and Martins, J. R. R. A., "Scalable parallel approach for high-fidelity steady-state aeroelastic analysis and adjoint derivative computations," AIAA Journal, Vol. 52, 2014, pp. 935-951.

${ }^{5}$ Palacios, F., Economon, T. D., Aranake, A. C., Copeland, S. R., Lonkar, A. K., Lukaczyk, T. W., Manosalvas, D. E., Naik, K. R., Padrón, A. S., Tracey, B., et al., "Stanford University Unstructured (SU2): Open-source analysis and design technology for turbulent flows," AIAA paper, Vol. 243, 2014, pp. 13-17.

${ }^{6}$ Kennedy, G. J. and Martins, J. R. R. A., "A parallel finite-element framework for large-scale gradient-based design optimization of high-performance structures," Finite Elements in Analysis and Design, Vol. 87, 2014, pp. 56 - 73.

${ }^{7}$ Liem, R. P., Kenway, G. K. W., and Martins, J. R. R. A., "Multimission Aircraft Fuel Burn Minimization via Multipoint Aerostructural Optimization," AIAA Journal, Vol. 53, 2015.

${ }^{8}$ Hwang, J. T., Roy, S., Kao, J. Y., Martins, J. R. R. A., and Crossley, W. A., "Simultaneous aircraft allocation and mission optimization using a modular adjoint approach," 56th AIAA SDM Conference, Kissimmee, FL, 2015.

${ }^{9}$ Jones, S., An Introduction to Thermodynamic Performance Analysis of Aircraft Gas Turbine Engine Cycles Using the Numerical Propulsion System Simulation Code, NASA, 2007, TM-2007-214690.

${ }^{10}$ Felder, J. L., Kim, H. D., and Brown, G. V., "Turboelectric distributed propulsion engine cycle analysis for hybrid-wingbody aircraft," 47th AIAA Aerospace Sciences Meeting, Orlando, FL, January, 2009.

${ }^{11}$ Freeh, J. E., Pratt, J. W., and Brouwer, J., "Development of a solid-oxide fuel cell/gas turbine hybrid system model for aerospace applications," ASME Turbo Expo 2004: Power for Land, Sea, and Air, American Society of Mechanical Engineers, 2004, pp. 371-379.

${ }^{12}$ Geiselhart, K. A., Ozoroski, L. P., Fenbert, J. W., Shields, E. W., and Li, W., "Integration of multifidelity multidisciplinary computer codes for design and analysis of supersonic aircraft," 49th AIAA Aerospace Sciences Meeting, No. 2011-465, 2011.

${ }^{13}$ Hendricks, E. S., Jones, S. M., and Gray, J. S., "Design Optimization of a Variable-Speed Power-Turbine," $50 T H$ AIAA/ASME/SAE/ASEE Joint Propulsion Conference, AIAA, Cleveland, Ohio, July 2014, AIAA-2014-3445.

${ }^{14}$ Gray, J. S., Chin, J. C., Hendricks, E. S., Hearn, T. A., and Lavelle, T., "Thermodynamics For Gas Turbine Cycles With Analytic Derivatives in OpenMDAO," 2016 AIAA SciTech Conference, American Institute of Aeronautics and Astronautics, Janurary 2016.

${ }^{15}$ Martins, J. R. R. A. and Hwang, J. T., "Review and Unification of Methods for Computing Derivatives of Multidisciplinary Computational Models," AIAA Journal, Vol. 51, No. 11, November 2013, pp. 2582-2599.

${ }^{16}$ Saad, Y. and Schultz, M. H., "GMRES: A generalized minimal residual algorithm for solving nonsymmetric linear systems," SIAM Journal on scientific and statistical computing, Vol. 7, No. 3, 1986, pp. 856-869.

${ }^{17}$ Haftka, R. T., "Simultaneous analysis and design," AIAA journal, Vol. 23, No. 7, 1985, pp. 1099-1103.

${ }^{18}$ Jones, S. M., "Steady-State Modeling of Gas Turbine Engines Using The Numerical Propulsion System Simulation Code," ASME Turbo Expo 2010: Power for Land, Sea, and Air, American Society of Mechanical Engineers, 2010, pp. 89-116.

${ }^{19}$ Consortium, N. P. S. S. et al., "NPSS User Guide, Software Release: NPSS 2.3. 1, Revision 2," User Documentation.

${ }^{20}$ Gill, P. E., Murray, W., and Saunders, M. A., "SNOPT: An SQP algorithm for large-scale constrained optimization," SIAM journal on optimization, Vol. 12, No. 4, 2002, pp. 979-1006. 